\title{
Association between Green Tea Consumption and Abdominal Obesity Risk in Middle-Aged Korean Population: Findings from the Korean Genome and Epidemiology Study
}

\author{
Junkyung Kwak (D) and Dayeon Shin *D
}

Citation: Kwak, J.; Shin, D. Association between Green Tea Consumption and Abdominal Obesity Risk in Middle-Aged Korean Population: Findings from the Korean Genome and Epidemiology Study. Int. J. Environ. Res. Public Health 2022, 19, 2735. https:// doi.org/10.3390/ijerph19052735

Received: 23 December 2021 Accepted: 18 February 2022 Published: 26 February 2022

Publisher's Note: MDPI stays neutral with regard to jurisdictional claims in published maps and institutional affiliations.

Copyright: (C) 2022 by the authors. Licensee MDPI, Basel, Switzerland. This article is an open access article distributed under the terms and conditions of the Creative Commons Attribution (CC BY) license (https:/ / creativecommons.org/licenses/by/ $4.0 /)$.
Department of Food and Nutrition, Inha University, Incheon 22212, Korea; oppo9012@naver.com

* Correspondence: dyshin@inha.ac.kr

\begin{abstract}
The prevalence of general and abdominal obesity is increasing with rapid economic growth and the westernization of dietary habits in Korea, especially in the middle-aged population. Data were obtained from the Korean Genome and Epidemiology Study (KoGES), which recruited 10,030 participants between the ages of 40 and 69 years. Information on green tea consumption was obtained from the food frequency questionnaire and categorized as none, $<1$ cup, between 1 and $<4$ cups, and $\geq 4$ cups. Multivariable logistic regression models were used to estimate the ORs and $95 \%$ CIs to examine any possible associations between green tea consumption and the risk of abdominal obesity after controlling for potential confounders. High consumption of green tea was associated with a $44 \%$ lower odds ratio for abdominal obesity (none vs. $\geq 4$ cups/week: OR, 0.56 ; $95 \%$ CI 0.41-0.78; $p$ for trend $=0.001$ ). When stratified by sex, an inverse association between green tea consumption and abdominal obesity was observed only in women (none vs. $\geq 4$ cups/week: OR, $0.71 ; 95 \%$ CI 0.57-0.88; $p$ for trend $=0.004$ ). No significant association was found among men. Our findings indicate that green tea consumption has beneficial effects in the prevention of abdominal obesity in middle-aged Korean women.
\end{abstract}

Keywords: metabolic syndrome; abdominal obesity; green tea consumption; Korean Genome and Epidemiology Study (KoGES)

\section{Introduction}

Abdominal obesity is a metabolic syndrome that is highly associated with factors that significantly increase the risk of cardiovascular disease (CVD) [1]. According to the Statistical Yearbook of Health Examinations published in 2019 by the National Health Insurance Corporation, the overall prevalence of abdominal obesity in Koreans is $24.8 \%$ (29.5\% in men and $19.8 \%$ in women). The overall prevalence of abdominal obesity of people under the age of 20 was $15.3 \%$ (22.0\% in men and $8.4 \%$ in women), whereas the overall prevalence among people in their 50 s was $23.4 \%(28.2 \%$ in men and $18.6 \%$ in women) [2]. According to a survey, the prevalence of abdominal obesity was approximately 10\% higher in those aged 40-69 years. Abdominal obesity was independently associated with CVD, myocardial infarction, and total mortality [3-5]. An effective reduction in waist circumference results from reduced abdominal fat and body fat-related risk rates, such as serum lipids [1,6]. Abdominal fat is influenced by environmental factors such as physical activity, genetic factors, sex, hormones, stress, and dietary quality, which are risk factors for abdominal obesity [7,8]. Many studies have shown that the dietary factors from among these various risk factors are particularly significant and directly contribute to the abdominal obesity rates [9-11].

Green tea is mainly consumed in Asia and has various pharmacological effects, including the treatment and prevention of obesity, particularly because it is associated with increased fatty acid oxidation and reduces body fat and abdominal fat [6,12-15]. An experimental epidemiological study conducted in Japan reported that weight gain and fat tissue 
in the abdominal cavity were significantly suppressed in the diet groups containing $2 \%$ and $4 \%$ powdered green tea as a result of supplying a diet containing $1 \%, 2 \%$, and $4 \%$ powdered green tea [16]. Furthermore, an analysis of total cholesterol in the liver, neutrophils in serum and liver, free fatty acid (FFA) in serum, and leptin levels showed that the powdered green tea group had lower concentrations than the control group in 4-week-old ICR female mice (18 g) [16]. Another experimental study conducted in Japan reported that consumption of green tea catechins (625 mg/day) enhanced exercise-induced loss of abdominal fat and was associated with decreased triglyceride levels and the circulating FFA in overweight and obese adults [17]. An experimental epidemiological study conducted in China reported that incubation with green tea polyphenols (GTPs) promoted catabolism of abdominal adipose tissue and was associated with increased lipid metabolism in the skeletal muscle of male chickens (35 days old) [18]. A randomized, placebo-controlled study conducted in Shanghai reported that daily consumption of 500-900 mg of green tea catechin with less than $200 \mathrm{mg}$ of caffeine can have a positive effect on reducing abdominal fat, particularly in Asian and overweight subjects [19]. Several animal and human studies reported that the groups that consumed green tea were significantly associated with decreased blood glucose, total cholesterol, LDL cholesterol levels, and triglycerides while they were associated with increased HDL cholesterol levels compared to the control group [14,15,20,21].

In Korea, with rapid economic growth and westernization of dietary habits, the prevalence of abdominal and general obesity is increasing, especially in the middle-aged population. However, most studies have been conducted on the role of green tea consumption in general obesity, and few studies have focused on the prevalence of abdominal obesity in Korean adults. In this study, the association between green tea consumption and abdominal obesity was examined using data derived from the Korean Genome and Epidemiology Study (KoGES) cohort of middle-aged Korean adults. Furthermore, the characteristics of the population, based on abdominal obesity and green tea consumption, were investigated. Abdominal obesity is a component of metabolic syndrome and is known to be related to other biomarkers such as blood pressure, fasting blood glucose, and blood lipid levels [1]; therefore, this study analyzed it with blood indicators related to abdominal obesity.

\section{Materials and Methods}

\subsection{Study Participants}

The KoGES, a large, population-based prospective cohort study, targeted Koreans with chronic diseases such as hypertension, obesity, cardiovascular disease, metabolic syndrome and type 2 diabetes [22]. It was designed to identify genetic and environmental factors and their interrelationships [22]. Since 2001, studies have been conducted in the Ansan and Ansung regions of South Korea on the KoGES. The Ansung cohort consisted of a rural population, while the Ansan cohort was a medium-sized city population.

In brief, 10,030 participants were enrolled between the ages of 40 and 69 (5012 from a farming community in Ansung and 5018 from an urban community in Ansan). Of these 10,030 participants, those who did not provide information on waist circumference measurements and green tea consumption were excluded $(n=256,2.6 \%)$. Among the remaining 9774 participants, those with missing information on area, sex, age, BMI, smoking status, and alcohol consumption were excluded $(n=2316)$. Among the remaining 7458 subjects, participants with missing information on fiber intake, carbohydrate intake, fat intake, protein intake, and energy intake were excluded $(n=48)$. Among the remaining 7410 subjects, participants who had missing information were excluded $(n=159)$. Among the remaining 7251 subjects, those with a history of cancer and CVD (treatment history, drug history, and medical history of hypertension, coronary artery disease, congestive heart failure, myocardial infarction and hyperlipidemia) were excluded $(n=1373)$ [23]. The final analytic sample size consisted of 5878 subjects ( 2875 men and 3003 women) according to the exclusion criteria of study participants (Figure 1) [24]. 


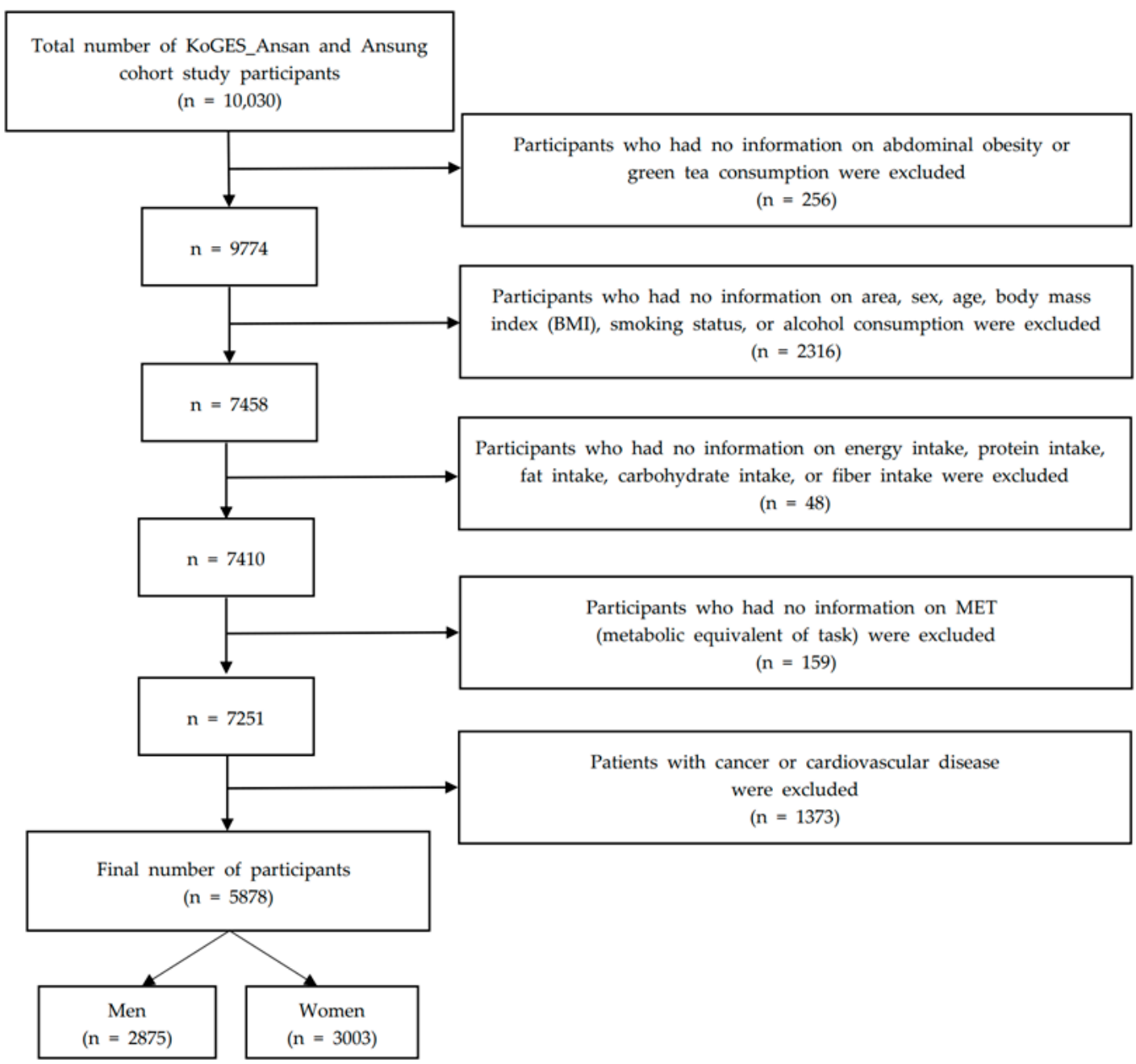

Figure 1. Process flow chart outlining the relevant steps for this analysis.

\subsection{Assessment of Abdominal Obesity}

Waist circumference was measured using standard methods with light clothing. It was calculated as the average of measurements over three times with the perimeter horizontal, the intermediate point between the rib (rib bone) and the iliac ridge. The average of measurements was read up to one decimal point in centimeters $(\mathrm{cm})$. Abdominal obesity is defined as at least $90 \mathrm{~cm}$ around the waist of a man and, $80 \mathrm{~cm}$ around the waist of a woman, as defined by the amended National Cholesterol Educational Program Adult Treatment Panel III (NCEP-ATP III) [1].

\subsection{Assessment of Green Tea Consumption}

The participants' green tea intake was estimated based on a semi-quantitative food frequency questionnaire (FFQ) comprising 106 food items. This questionnaire was developed to determine the relationship between dietary intake and disease among Koreans [25]. Using a questionnaire based on foods frequently consumed by Koreans aged 40-69 years, a food list and reference amount were presented to investigate the frequency and amount of intake of each food, and various auxiliary tools were used so that the subjects could accurately respond to the amount of food consumed. The participants' usual green tea intake was investigated using a semi-quantitative FFQ during the past year. Follow-up was conducted to complete the FFQ survey periodically by well-trained interviewers. Green tea consumption was surveyed based on a green tea bag sold in the market. The amount of green tea served $(120 \mathrm{~mL})$ was set based on the reference amount suggested by the KoGES. Green tea intake in the questionnaire was classified according to the frequency of 
intake (1-2 times a day, 3-4 times a day, and 5 times a day) and average intake per serving ( $1 / 2$ cup, 1 cup, and 2 cups). In this study, green tea intake was classified according to frequency (none, $<1$ cup, between 1 and $<4$ cups, and $\geq 4$ cups) on a weekly basis, and the portion size was set at $120 \mathrm{~mL}$ per cup based on the KoGES FFQ guideline [26].

\subsection{Assessment of Other Variables}

KoGES research data collection was conducted only for participants who showed a willingness to participate in the study. To determine the demographic information, lifestyle, medical history, and health conditions of the study participants, a survey and examination were conducted for $2-3 \mathrm{~h}$ at the site. Blood and urine were used for biochemical tests to diagnose health conditions, and the rest were separated according to their components (serum, plasma, DNA, etc.) and stored at the Korea Centers for Disease Control and Prevention.

Education level was classified as elementary school or lower, middle/high school, and college or higher. Household income was categorized as <100, 100-200, 200-300, and $>300$ (10,000 won/month). The MET, which is based on physical activity during the day, was classified as follows: sedentary lifestyle, low-intensity activity, moderate-intensity activity, and high-intensity activity. Specifically, sedentary life included typing, driving, office work, playing, sewing class, ironing, writing, and cooking; low-intensity activities included walking, cleaning, laundry, babysitting, bathing, and exercise as entertainment (bicycle, table tennis); medium-intensity activities included fast walking, woodwork, lawn mowing, snow removal, and regular exercise (badminton, swimming, tennis); and highintensity activities included sports, mountaineering, running, tree cutting, agriculture, forestry, and mining. After entering different values according to exercise intensity, the MET was calculated by changing the existing units of minutes per day to hours per day.

The follow-up participants regularly attended community clinics for anthropometric measurements and biochemical tests (including fasting blood glucose, fasting insulin, Creactive protein $(\mathrm{CRP})$, blood pressure, blood lipid levels, waist circumference, weight, and height) performed by trained healthcare providers. BMI was calculated as the weight divided by height squared $\left(\mathrm{kg} / \mathrm{m}^{2}\right)$ and was measured using tetrapolar bioelectrical impedance analysis (InBody version 3.0; InBody, Seoul, Korea). After overnight fasting for $12 \mathrm{~h}$, the plasma concentrations of total cholesterol, HDL cholesterol, glucose and triglycerides were assessed using an immunoradiometric assay (ADVIA 1650; Siemens, Tarrytown, NY, USA). Systolic and diastolic blood pressures were measured on the left and right arms with the subjects in a sitting position in a stable state, and their average values were calculated. When measuring blood pressure, the arm and back were supported, and blood pressure was assessed in a sitting posture with the arm held at the heart level.

In the FFQ, the intake of nutrients of each food item was calculated, taking into account the weight caused by the portion size of each food item and the frequency of intake. The sum of the nutrients consumed by each food item determines the daily nutrient intake of the individual. Total energy intake was calculated in kcal per day (kcal/day). Carbohydrate intake, protein intake, fat intake, and fiber intake were calculated in grams per day (g/day).

\subsection{Statistical Analyses}

All data are expressed as mean and standard deviation or as numbers and percentages for descriptive statistics. The general characteristics of the participants according to abdominal obesity were compared using the chi-squared test for categorical variables and $t$-test for continuous variables. The general characteristics of the participants according to weekly green tea consumption were compared using chi-squared tests for categorical variables and ANOVA for continuous variables.

The relationship between green tea consumption and abdominal obesity was examined using multiple logistic regression models. Unadjusted and adjusted odds ratios (ORs) and 95\% confidence intervals [27] were calculated to assess the association between green tea consumption and abdominal obesity. The $p$-value for trend was calculated using 
the median for each category of weekly green tea consumption as a continuous variable. Adjustments were made for age, sex, area, alcohol consumption, smoking, BMI, education level, household income, MET, energy intake, protein intake, fat intake, carbohydrate intake, and fiber intake. We further analyzed the effects of green tea consumption by sex because the prevalence of abdominal obesity and reported risk factors differ by sex. All statistical analyses were performed using IBM SPSS (Statistical Package for Social Science) Statistics 26 (IBM, Armonk, NY, USA). Statistical significance was set at $p<0.05$.

\section{Results}

\subsection{Associations of Abdominal Obesity with Other Risk Factors}

Table 1 describes the general characteristics of the participants according to sex and abdominal obesity (women with abdominal obesity, $\mathrm{n}=1367$; women without abdominal obesity, $\mathrm{n}=1636$; men with abdominal obesity, $\mathrm{n}=520$; men without abdominal obesity, $\mathrm{n}=2355)$.

Table 1. General characteristics of study participants by abdominal obesity in Korean Genome and Epidemiology Study (KoGES).

\begin{tabular}{|c|c|c|c|c|c|c|}
\hline \multirow[t]{2}{*}{ Variable } & \multicolumn{3}{|c|}{ Men $(n=2875)$} & \multicolumn{3}{|c|}{ Women $(n=3003)$} \\
\hline & $\begin{array}{l}\text { Abdominal } \\
\text { Obesity }\end{array}$ & $\begin{array}{c}\text { No Abdominal } \\
\text { Obesity }\end{array}$ & $p$-Value ${ }^{1}$ & $\begin{array}{l}\text { Abdominal } \\
\text { Obesity }\end{array}$ & $\begin{array}{l}\text { No Abdominal } \\
\text { Obesity }\end{array}$ & $p$-Value ${ }^{1}$ \\
\hline Participants, $\mathrm{n}$ & 520 & 2355 & & 1367 & 1636 & \\
\hline Age (years) & $50.5 \pm 8.2$ & $49.9 \pm 8.3$ & 0.178 & $53.5 \pm 8.8$ & $48.3 \pm 7.7$ & $<0.0001$ \\
\hline Area & & & 0.009 & & & $<0.0001$ \\
\hline Ansung & $185(35.6 \%)$ & $700(29.7 \%)$ & & $711(52.0 \%)$ & $349(21.3 \%)$ & \\
\hline Ansan & $335(64.4 \%)$ & $1655(70.3 \%)$ & & $656(48.0 \%)$ & $1287(78.7 \%)$ & \\
\hline Education level & & & 0.705 & & & $<0.0001$ \\
\hline Elementary school or lower & $80(15.4 \%)$ & $394(16.7 \%)$ & & $710(51.9 \%)$ & $420(25.7 \%)$ & \\
\hline Middle/High school & $320(61.5 \%)$ & $1409(59.8 \%)$ & & $609(44.6 \%)$ & $1040(63.6 \%)$ & \\
\hline College or higher & $120(23.1 \%)$ & $552(23.4 \%)$ & & $48(3.5 \%)$ & $176(10.8 \%)$ & \\
\hline Household income (10,000 won/month) & & & 0.297 & & & $<0.0001$ \\
\hline$<100$ & $114(21.9 \%)$ & $523(22.2 \%)$ & & $634(46.4 \%)$ & $379(23.2 \%)$ & \\
\hline $100-200$ & $147(28.3 \%)$ & $693(29.4 \%)$ & & $376(27.5 \%)$ & $500(30.6 \%)$ & \\
\hline $200-300$ & $105(20.2 \%)$ & $523(22.6 \%)$ & & $203(14.9 \%)$ & $393(24.0 \%)$ & \\
\hline$\geq 300$ & $154(29.6 \%)$ & $607(25.8 \%)$ & & $154(11.3 \%)$ & $364(22.2 \%)$ & \\
\hline Body mass index $\left(\mathrm{kg} / \mathrm{m}^{2}\right)$ & $27.6 \pm 2.0$ & $23.4 \pm 2.4$ & $<0.0001$ & $26.5 \pm 2.9$ & $22.9 \pm 2.3$ & $<0.0001$ \\
\hline Alcohol consumption & & & 0.295 & & & $<0.0001$ \\
\hline None & $86(16.5 \%)$ & $437(18.6 \%)$ & & $976(71.4 \%)$ & $1107(67.7 \%)$ & \\
\hline Past & $54(10.4 \%)$ & $203(8.6 \%)$ & & $54(4.0 \%)$ & $30(1.8 \%)$ & \\
\hline Current & $380(73.1 \%)$ & $1715(72.8 \%)$ & & $337(24.7 \%)$ & $499(30.5 \%)$ & \\
\hline Smoking status & & & 0.122 & & & 0.363 \\
\hline None & $114(21.9 \%)$ & $458(19.4 \%)$ & & $1297(94.9 \%)$ & $1557(95.2 \%)$ & \\
\hline Past & $167(32.1 \%)$ & $699(29.7 \%)$ & & $22(1.6 \%)$ & $17(1.0 \%)$ & \\
\hline Current & $239(46.0 \%)$ & $1198(50.9 \%)$ & & $48(3.5 \%)$ & $62(3.8 \%)$ & \\
\hline MET (hours/day) $^{2}$ & $23.6 \pm 14.8$ & $24.7 \pm 15.7$ & 0.017 & $23.7 \pm 14.8$ & $20.1 \pm 11.8$ & $<0.0001$ \\
\hline \multicolumn{7}{|l|}{ Dietary intake } \\
\hline Total energy intake (kcal/day) & $2124.6 \pm 726.7$ & $1999.9 \pm 620.4$ & $<0.0001$ & $1935.0 \pm 730.7$ & $1863.9 \pm 636.0$ & 0.005 \\
\hline Protein intake (g/day) & $75.2 \pm 32.5$ & $69.4 \pm 28.1$ & $<0.0001$ & $64.7 \pm 29.8$ & $65.0 \pm 30.0$ & 0.761 \\
\hline Fat intake (g/day) & $39.4 \pm 22.7$ & $36.5 \pm 21.2$ & 0.008 & $29.5 \pm 19.5$ & $32.0 \pm 20.5$ & 0.001 \\
\hline Carbohydrate intake (g/day) & $361.8 \pm 115.9$ & $343.1 \pm 96.7$ & 0.001 & $348.6 \pm 128.7$ & $324.9 \pm 103.3$ & $<0.0001$ \\
\hline Fiber intake (g/day) & $7.3 \pm 3.5$ & $6.8 \pm 3.1$ & 0.001 & $7.3 \pm 3.8$ & $6.8 \pm 3.2$ & $<0.0001$ \\
\hline Weekly green tea consumption (cup) & $2.7 \pm 5.0$ & $2.4 \pm 4.2$ & 0.247 & $1.8 \pm 4.3$ & $2.6 \pm 4.9$ & $<0.0001$ \\
\hline \multicolumn{7}{|l|}{ Blood pressure $(n=5872)$} \\
\hline Systolic blood pressure (mmHg) & $123.3 \pm 15.7$ & $118.0 \pm 15.3$ & $<0.0001$ & $120.2 \pm 16.6$ & $111.7 \pm 16.2$ & $<0.0001$ \\
\hline Diastolic blood pressure (mmHg) & $84.0 \pm 10.1$ & $79.7 \pm 10.4$ & $<0.0001$ & $78.9 \pm 10.6$ & $73.5 \pm 10.2$ & $<0.0001$ \\
\hline
\end{tabular}


Table 1. Cont.

\begin{tabular}{|c|c|c|c|c|c|c|}
\hline \multirow[t]{2}{*}{ Variable } & \multicolumn{3}{|c|}{$\operatorname{Men}(n=2875)$} & \multicolumn{3}{|c|}{ Women $(n=3003)$} \\
\hline & $\begin{array}{l}\text { Abdominal } \\
\text { Obesity }\end{array}$ & $\begin{array}{l}\text { No Abdominal } \\
\text { Obesity }\end{array}$ & $p$-Value ${ }^{1}$ & $\begin{array}{l}\text { Abdominal } \\
\text { Obesity }\end{array}$ & $\begin{array}{c}\text { No Abdominal } \\
\text { Obesity }\end{array}$ & $p$-Value ${ }^{1}$ \\
\hline \multicolumn{7}{|l|}{ Biomarkers } \\
\hline Fasting blood glucose $(\mathrm{mg} / \mathrm{dL})(\mathrm{n}=5785)$ & $94.2 \pm 24.8$ & $89.2 \pm 23.4$ & $<0.0001$ & $86.6 \pm 23.1$ & $81.7 \pm 14.1$ & $<0.0001$ \\
\hline Total cholesterol $(\mathrm{mg} / \mathrm{dL})(\mathrm{n}=5878)$ & $199.5 \pm 34.5$ & $191.2 \pm 36.1$ & $<0.0001$ & $194.6 \pm 37.2$ & $184.9 \pm 32.9$ & $<0.0001$ \\
\hline HDL cholesterol $(\mathrm{mg} / \mathrm{dL})^{3}(\mathrm{n}=5878)$ & $40.1 \pm 7.5$ & $44.5 \pm 10.0$ & $<0.0001$ & $44.0 \pm 9.4$ & $48.4 \pm 10.4$ & $<0.0001$ \\
\hline Triglyceride $(\mathrm{mg} / \mathrm{dL})(\mathrm{n}=5878)$ & $215.5 \pm 135.3$ & $163.2 \pm 107.4$ & $<0.0001$ & $159.6 \pm 97.9$ & $122.0 \pm 63.4$ & $<0.0001$ \\
\hline $\mathrm{CRP}(\mathrm{mg} / \mathrm{dL})^{4}(\mathrm{n}=5877)$ & $0.28 \pm 0.40$ & $0.22 \pm 0.41$ & 0.004 & $0.23 \pm 0.38$ & $0.17 \pm 0.42$ & $<0.0001$ \\
\hline Fasting insulin $(\mathrm{uIU} / \mathrm{mL})(\mathrm{n}=5786)$ & $8.9 \pm 5.5$ & $6.5 \pm 3.5$ & $<0.0001$ & $8.7 \pm 6.2$ & $7.0 \pm 4.3$ & $<0.0001$ \\
\hline
\end{tabular}

Data are presented as the mean \pm standard deviation (SD) or number (\%). ${ }^{1} p$-values were calculated using the chi-squared test for categorical variables and $t$-test for continuous variables. ${ }^{2}$ MET, metabolic equivalent of task

${ }^{3}$ HDL cholesterol, high-density lipoprotein cholesterol. ${ }^{4} \mathrm{CRP}, \mathrm{C}$-reactive protein.

Regardless of sex, the abdominal obesity group had a higher proportion of residents in Ansung, BMI $\left(\mathrm{kg} / \mathrm{m}^{2}\right)$, fasting blood glucose $(\mathrm{mg} / \mathrm{dL})$, total cholesterol $(\mathrm{mg} / \mathrm{dL})$, triglyceride levels $(\mathrm{mg} / \mathrm{dL}), \mathrm{CRP}(\mathrm{mg} / \mathrm{dL})$, fasting insulin $(\mathrm{uIU} / \mathrm{mL})$, average systolic blood pressure $(\mathrm{mmHg})$, average diastolic blood pressure $(\mathrm{mmHg})$, total energy intake ( $\mathrm{kcal} /$ day), carbohydrate intake (g/day), and fiber intake (g/day) and lower HDL cholesterol (mg/dL) compared to the non-abdominal obesity group (all $p$ values $<0.05$ ). In women, the abdominal obesity group had a higher mean age, MET (hours/day), the proportion of education level of elementary school or lower, income level less than 100 (10,000 won/month), and non-drinkers and lower fat intake ( $\mathrm{g} /$ day) compared to the non-abdominal obesity group (all $p$ values $<0.05$ ). Among men, the abdominal obesity group had higher protein intake (g/day) and fat intake (g/day) and lower MET (hours/day) than the non-abdominal obesity group (all $p$ values $<0.05$ ). Regarding the relationship between abdominal obesity and weekly green tea consumption (in cups), weekly green tea consumption was higher in the non-abdominal obesity group than in the abdominal obesity group only in women $(p<0.0001 ; 1.8 \pm 4.3$ vs. $2.6 \pm 4.9)$.

\subsection{Associations of Green Tea Consumption per Week with Other Risk Factors}

Table 2 describes the participants' general characteristics according to sex and weekly green tea consumption (none, $\mathrm{n}=2236 ;<1$ cup $/$ week, $\mathrm{n}=1058 ; 1$ to $<4$ cups $/$ week, $\mathrm{n}=1501$; $\geq 4$ cups /week, $\mathrm{n}=1083$ ).

Regardless of sex, frequent green tea drinkers ( $\geq 4$ cups/week) had lower mean age, MET, proportion of residents in Ansung, education level of elementary school or lower, income level less than 100 (10,000 won/month), average systolic blood pressure ( $\mathrm{mmHg}$ ), total energy intake ( $\mathrm{kcal} /$ day), protein intake, fat intake ( $\mathrm{g} /$ day), carbohydrate intake (g/day), and fiber intake (g/day) compared with non-drinkers (all $p$ values $<0.05$ ). In women, frequent green tea drinkers ( $\geq 4$ cups/week) had a lower proportion of nondrinkers, total cholesterol $(\mathrm{mg} / \mathrm{dL})$, HDL cholesterol $(\mathrm{mg} / \mathrm{dL})$, and average diastolic blood pressure $(\mathrm{mmHg})$ than non-drinkers (all $p$ values $<0.05)$. In men, frequent green tea drinkers ( $\geq 4$ cups/week) had higher BMI, proportion of non-smokers, fasting blood glucose $(\mathrm{mg} / \mathrm{dL})$, and total cholesterol $(\mathrm{mg} / \mathrm{dL}$ ) than non-drinkers (all $p$ values $<0.0001)$. 
Table 2. General characteristics, biomarkers, and dietary intake of the KoGES Cohort Study participants by weekly green tea consumption (no. of cups)

\begin{tabular}{|c|c|c|c|c|c|c|c|c|c|c|}
\hline \multirow{3}{*}{ Variable } & \multicolumn{5}{|c|}{ Men $(n=2875)$} & \multicolumn{5}{|c|}{ Women $(n=3003)$} \\
\hline & \multicolumn{10}{|c|}{ Weekly Green Tea Consumption (No. of Cups) } \\
\hline & None & $<1$ & 1 to $<4$ & $\geq 4$ & $p$-Value ${ }^{1}$ & None & $<1$ & 1 to $<4$ & $\geq 4$ & $p$-Value ${ }^{1}$ \\
\hline Participants, $\mathrm{n}$ & 1099 & 483 & 727 & 566 & & 1137 & 575 & 774 & 517 & \\
\hline Age (years) & $52.0 \pm 8.9$ & $50.3 \pm 8.5$ & $49.1 \pm 7.7$ & $47.1 \pm 6.5$ & $<0.0001$ & $53.1 \pm 8.9$ & $50.7 \pm 8.6$ & $48.7 \pm 7.9$ & $47.9 \pm 7.5$ & $<0.0001$ \\
\hline Area & & & & & $<0.0001$ & & & & & $<0.0001$ \\
\hline Ansung & $439(39.9 \%)$ & $185(38.3 \%)$ & $178(24.5 \%)$ & $83(14.7 \%)$ & & $557(49.0 \%)$ & $238(41.4 \%)$ & $168(21.7 \%)$ & $97(18.8 \%)$ & \\
\hline Ansan & $660(60.1 \%)$ & $298(61.7 \%)$ & $549(75.5 \%)$ & $483(85.3 \%)$ & & $580(51.0 \%)$ & $337(58.6 \%)$ & $606(78.3 \%)$ & $420(81.2 \%)$ & \\
\hline Elementary school or lower & $276(25.1 \%)$ & $85(17.6 \%)$ & $80(11.0 \%)$ & $33(5.8 \%)$ & & $611(53.7 \%)$ & $223(38.8 \%)$ & $196(25.3 \%)$ & $100(19.3 \%)$ & \\
\hline Middle/High school & $681(62.0 \%)$ & $303(62.7 \%)$ & $449(61.8 \%)$ & $296(52.3 \%)$ & & $478(42 \%)$ & $317(55.1 \%)$ & $496(64.1 \%)$ & $358(69.2 \%)$ & \\
\hline College or higher & $142(12.9 \%)$ & $95(19.7 \%)$ & $198(27.2 \%)$ & $237(41.9 \%)$ & & $48(4.2 \%)$ & $35(6.1 \%)$ & $82(10.6 \%)$ & $59(11.4 \%)$ & \\
\hline Household income (10,000 won/month) & & & & & $<0.0001$ & & & & & $<0.0001$ \\
\hline$<100$ & $357(32.5 \%)$ & $122(25.3 \%)$ & $108(14.9 \%)$ & $50(8.8 \%)$ & & $528(46.4 \%)$ & $215(37.4 \%)$ & $177(22.9 \%)$ & $93(18.0 \%)$ & \\
\hline $100-200$ & $336(30.6 \%)$ & $166(34.4 \%)$ & $219(30.1 \%)$ & $119(21.0 \%)$ & & $311(27.4 \%)$ & $185(32.2 \%)$ & $224(28.9 \%)$ & $156(30.2 \%)$ & \\
\hline 200-300 & $211(19.2 \%)$ & $100(20.7 \%)$ & $172(23.7 \%)$ & $154(27.2 \%)$ & & $178(15.7 \%)$ & $95(16.5 \%)$ & $205(26.5 \%)$ & $118(22.8 \%)$ & \\
\hline$\geq 300$ & $195(17.7 \%)$ & $95(19.7 \%)$ & $228(31.4 \%)$ & $243(42.9 \%)$ & & $120(10.6 \%)$ & $80(13.9 \%)$ & $168(21.7 \%)$ & $150(29.0 \%)$ & \\
\hline Body mass index $\left(\mathrm{kg} / \mathrm{m}^{2}\right)$ & $23.8 \pm 3.0$ & $23.9 \pm 2.9$ & $24.4 \pm 2.7$ & $24.7 \pm 2.5$ & $<0.0001$ & $24.5 \pm 3.3$ & $24.4 \pm 3.1$ & $24.5 \pm 3.1$ & $24.6 \pm 3.0$ & 0.745 \\
\hline None & $208(18.9 \%)$ & $98(20.3 \%)$ & $118(16.2 \%)$ & $99(17.5 \%)$ & & $833(73.3 \%)$ & $405(70.4 \%)$ & $495(64.0 \%)$ & $350(67.7 \%)$ & \\
\hline Past & $108(9.8 \%)$ & $44(9.1 \%)$ & $66(9.1 \%)$ & $39(6.9 \%)$ & & $35(3.1 \%)$ & $18(3.1 \%)$ & $18(2.3 \%)$ & $13(2.5 \%)$ & \\
\hline Current & $783(71.2 \%)$ & $341(70.6 \%)$ & $543(74.7 \%)$ & $428(75.6 \%)$ & & $269(23.7 \%)$ & $152(26.4 \%)$ & $261(33.7 \%)$ & $154(29.8 \%)$ & \\
\hline Smoking status & & & & & $<0.0001$ & & & & & 0.382 \\
\hline None & $171(15.6 \%)$ & $110(22.8 \%)$ & $178(24.5 \%)$ & $113(20.0 \%)$ & & 1075 (94.5\%) & $545(94.8 \%)$ & $747(96.5 \%)$ & $487(94.2 \%)$ & \\
\hline Past & $283(25.8 \%)$ & $147(30.4 \%)$ & $243(33.4 \%)$ & $193(34.1 \%)$ & & $17(1.5 \%)$ & $7(1.2 \%)$ & $9(1.2 \%)$ & $6(1.2 \%)$ & \\
\hline Current & $645(58.7 \%)$ & $226(46.8 \%)$ & $306(42.1 \%)$ & $260(45.9 \%)$ & & $45(4.0 \%)$ & $23(4.0 \%)$ & $18(2.3 \%)$ & $24(4.6 \%)$ & \\
\hline MET (hours/day) ${ }^{2}$ & $24.7 \pm 15.7$ & $23.6 \pm 14.8$ & $22.4 \pm 13.3$ & $20.3 \pm 11.9$ & $<0.0001$ & $24.0 \pm 15.0$ & $21.4 \pm 13.8$ & $20.4 \pm 11.8$ & $19.0 \pm 10.5$ & $<0.0001$ \\
\hline \multicolumn{11}{|l|}{ Dietary intake } \\
\hline Total energy intake (kcal/day) & $1972.8 \pm 677.9$ & $1912.5 \pm 541.4$ & $2039.4 \pm 560.5$ & $2191.1 \pm 714.0$ & $<0.0001$ & $1825.6 \pm 659.5$ & $1850.6 \pm 726.4$ & $1969.0 \pm 645.0$ & $1993.5 \pm 710.2$ & $<0.0001$ \\
\hline Protein intake (g/day) & $66.6 \pm 30.9$ & $63.9 \pm 24.5$ & $71.7 \pm 23.2$ & $81.7 \pm 32.1$ & $<0.0001$ & $59.7 \pm 28.8$ & $62.4 \pm 34.5$ & $68.9 \pm 25.5$ & $72.9 \pm 30.4$ & $<0.0001$ \\
\hline Fat intake (g/day) & $35.1 \pm 23.1$ & $32.9 \pm 19.2$ & $37.7 \pm 17.6$ & $43.3 \pm 23.4$ & $<0.0001$ & $27.5 \pm 19.2$ & $28.7 \pm 22.2$ & $33.9 \pm 16.9$ & $36.0 \pm 21.9$ & $<0.0001$ \\
\hline Carbohydrate intake (g/day) & $341.9 \pm 107.4$ & $334.4 \pm 84.3$ & $347.8 \pm 91.4$ & $363.7 \pm 109.1$ & $<0.0001$ & $330.1 \pm 113.7$ & $331.2 \pm 119.2$ & $343.1 \pm 114.9$ & $341.6 \pm 119.1$ & 0.048 \\
\hline Fiber intake (g/day) & $6.6 \pm 3.1$ & $6.4 \pm 3.2$ & $6.9 \pm 2.9$ & $7.6 \pm 3.5$ & $<0.0001$ & $6.7 \pm 3.4$ & $6.9 \pm 3.6$ & $7.3 \pm 3.3$ & $7.6 \pm 3.7$ & $<0.0001$ \\
\hline
\end{tabular}


Table 2. Cont.

\begin{tabular}{|c|c|c|c|c|c|c|c|c|c|c|}
\hline \multirow{3}{*}{ Variable } & \multicolumn{5}{|c|}{$\operatorname{Men}(n=2875)$} & \multicolumn{5}{|c|}{ Women $(n=3003)$} \\
\hline & \multicolumn{10}{|c|}{ Weekly Green Tea Consumption (No. of Cups) } \\
\hline & None & $<1$ & 1 to $<4$ & $\geq 4$ & $p$-Value ${ }^{1}$ & None & $<1$ & 1 to $<4$ & $\geq 4$ & $p$-Value ${ }^{1}$ \\
\hline \multicolumn{11}{|l|}{ Blood pressure $(n=5872)$} \\
\hline Systolic blood pressure $(\mathrm{mmHg})$ & $120.4 \pm 16.5$ & $119.6 \pm 15.7$ & $117.2 \pm 14.8$ & $117.9 \pm 13.7$ & $<0.0001$ & $118.1 \pm 17.6$ & $116.1 \pm 16.5$ & $113.5 \pm 16.2$ & $112.3 \pm 16.1$ & $<0.0001$ \\
\hline Diastolic blood pressure (mmHg) & $80.5 \pm 10.8$ & $80.3 \pm 10.5$ & $79.9 \pm 10.5$ & $81.0 \pm 9.8$ & 0.304 & $77.2 \pm 11.0$ & $76.6 \pm 10.6$ & $74.7 \pm 10.3$ & $74.3 \pm 10.6$ & $<0.0001$ \\
\hline \multicolumn{11}{|l|}{ Biomarkers } \\
\hline Fasting blood glucose $(\mathrm{mg} / \mathrm{dL})(\mathrm{n}=5785)$ & $88.2 \pm 21.4$ & $89.0 \pm 20.4$ & $90.5 \pm 22.7$ & $94.4 \pm 31.0$ & $<0.0001$ & $84.3 \pm 18.4$ & $83.0 \pm 18.6$ & $84.2 \pm 17.5$ & $83.7 \pm 22.0$ & 0.558 \\
\hline Total cholesterol $(\mathrm{mg} / \mathrm{dL})(\mathrm{n}=5878)$ & $189.7 \pm 36.2$ & $190.7 \pm 36.0$ & $194.1 \pm 34.9$ & $198.6 \pm 36.1$ & $<0.0001$ & $190.3 \pm 36.0$ & $185.7 \pm 34.2$ & $191.1 \pm 36.1$ & $188.5 \pm 33.3$ & 0.026 \\
\hline Triglyceride $(\mathrm{mg} / \mathrm{dL})(\mathrm{n}=5878)$ & $174.3 \pm 115.0$ & $168.8 \pm 116.6$ & $173.4 \pm 117.2$ & $171.8 \pm 109.5$ & 0.843 & $142.3 \pm 88.0$ & $142.8 \pm 81.9$ & $136.0 \pm 83.6$ & $132.5 \pm 71.2$ & 0.067 \\
\hline $\mathrm{CRP}(\mathrm{mg} / \mathrm{dL})^{4}(\mathrm{n}=5877)$ & $0.24 \pm 0.50$ & $0.22 \pm 0.33$ & $0.22 \pm 0.33$ & $0.25 \pm 0.38$ & 0.344 & $0.20 \pm 0.41$ & $0.21 \pm 0.46$ & $0.20 \pm 0.37$ & $0.18 \pm 0.33$ & 0.689 \\
\hline Fasting Insulin ( $\mathrm{IIU} / \mathrm{mL})(\mathrm{n}=5786)$ & $6.8 \pm 3.8$ & $7.0 \pm 4.3$ & $6.9 \pm 4.0$ & $7.2 \pm 4.4$ & 0.304 & $8.0 \pm 6.7$ & $7.7 \pm 4.2$ & $7.6 \pm 4.1$ & $7.6 \pm 4.7$ & 0.428 \\
\hline
\end{tabular}

Data are presented as the mean \pm standard deviation (SD) or number (\%). ${ }^{1} p$-values were calculated using the chi-squared test for categorical variables and ANOVA for continuous variables. ${ }^{2}$ MET, metabolic equivalent of task. ${ }^{3} \mathrm{HDL}$ cholesterol, high-density lipoprotein cholesterol. ${ }^{4} \mathrm{CRP}$, C-reactive protein. 


\subsection{Risk of Abdominal Obesity Due to Green Tea Consumption}

To assess the prevalence of abdominal obesity in relation to green tea consumption, multivariable logistic regression analysis was performed according to the level of green tea consumption per week to compute the adjusted odds ratio and 95\% CI, as shown in Table 3. We adjusted for age, sex, area, alcohol consumption, smoking, BMI, education level, household income, MET, energy intake, protein intake, fat intake, carbohydrate intake, and fiber intake. Compared with participants who did not drink green tea regardless of sex, crude ORs for the prevalence of abdominal obesity were 0.73 (95\% CI: 0.60-0.90), 0.52 (95\% CI: 0.43-0.63), and 0.45 (95\% CI: 0.36-0.56) for those drinking <1 cup/week, 1 to $<4$ cups/week, and $\geq 4$ cups/week, respectively.

Table 3. Odd ratios (OR) and 95\% confidence intervals of abdominal obesity by green tea consumption in KoGES Cohort Study.

\begin{tabular}{|c|c|c|c|c|c|}
\hline \multirow[b]{2}{*}{ (n = Cases/Total) } & \multicolumn{5}{|c|}{ Weekly Green Tea Consumption (No. of Cups) } \\
\hline & None & $<1$ & 1 to $<4$ & $\geq 4$ & $p$ for Trend $^{2}$ \\
\hline All $(\mathrm{n}=1887 / 5878)$ & $(\mathrm{n}=820 / 2236)$ & $(\mathrm{n}=354 / 1058)$ & $(\mathrm{n}=430 / 1501)$ & $(n=283 / 1083)$ & \\
\hline Crude OR $(95 \% \mathrm{CI})$ & 1.00 & $0.73(0.60-0.90)$ & $0.52(0.43-0.63)$ & $0.45(0.36-0.56)$ & $<0.0001$ \\
\hline Multivariable OR $(95 \% \mathrm{CI})^{1}$ & 1.00 & $0.89(0.67-1.18)$ & $0.74(0.56-0.98)$ & $0.56(0.41-0.78)$ & 0.001 \\
\hline $\operatorname{Men}(n=520 / 2875)$ & $(\mathrm{n}=200 / 1099)$ & $(\mathrm{n}=85 / 483)$ & $(\mathrm{n}=133 / 727)$ & $(\mathrm{n}=102 / 566)$ & \\
\hline Crude OR $(95 \%$ CI) & 1.00 & $0.96(0.73-1.27)$ & $1.01(0.79-1.28)$ & $0.99(0.76-1.29)$ & 0.987 \\
\hline Multivariable OR $(95 \% \mathrm{CI})^{1}$ & 1.00 & $0.85(0.56-1.29)$ & $0.92(0.64-1.31)$ & $0.82(0.54-1.24)$ & 0.439 \\
\hline Women $(n=1367 / 3003)$ & $(\mathrm{n}=620 / 1137)$ & $(\mathrm{n}=269 / 575)$ & $(\mathrm{n}=297 / 774)$ & $(\mathrm{n}=181 / 517)$ & \\
\hline Crude OR (95\% CI) & 1.00 & $0.92(0.80-1.05)$ & $0.72(0.64-0.81)$ & $0.65(0.56-0.74)$ & $<0.0001$ \\
\hline Multivariable OR (95\% CI) ${ }^{1}$ & 1.00 & $0.92(0.75-1.12)$ & $0.79(0.65-0.96)$ & $0.71(0.57-0.88)$ & 0.004 \\
\hline
\end{tabular}

${ }^{1}$ Adjusted for age, sex, area, alcohol consumption, smoking, body mass index, education level, household income, metabolic equivalent of task (MET), energy intake, protein intake, fat intake, carbohydrate intake, and fiber intake $2 p$ for trend was calculated using the median of weekly green tea consumption in each category.

After adjusting for confounding variables, multivariable ORs were significantly associated with a decrease for those drinking $<1$ cup/week (OR: 0.89; 95\% CI: 0.67-1.18), 1 to $<4$ cups/week (OR: 0.74; 95\% CI: 0.56-0.98), and $\geq 4$ cups/week (OR: 0.56; 95\% CI: 0.41-0.78) compared to those who did not drink green tea, regardless of sex. Based on the participants who did not consume green tea among women, participants that consumed 1 to $<4$ cups/week or $\geq 4$ cups/week of green tea had crude ORs (95\% CI) for abdominal obesity of $0.72(0.64-0.81)$ and $0.65(0.56-0.74)$, respectively. Furthermore, women who consumed 1 to $<4$ cups/week or $\geq 4$ cups/week of green tea had multivariable-adjusted OR $(95 \% \mathrm{CI})$ for abdominal obesity of $0.79(0.65-0.96)$ and $0.71(0.57-0.88)$, respectively, after adjusting for age, area, alcohol consumption, smoking, BMI, education level, household income, MET, energy intake, protein intake, fat intake, carbohydrate intake, and fiber intake. When stratified by sex, the degree of the association between green tea consumption and the prevalence of abdominal obesity in women tended to be significantly lower.

\section{Discussion}

In this study, we investigated the beneficial effects of green tea consumption on abdominal obesity according to sex, using community-based cohort data of middle-aged Korean adults. We found a significant negative correlation between the total population and women, but not for men as a result of studying the associations between abdominal obesity and green tea consumption.

In Ansung, a rural area, the proportion of participants who did not consume green tea was the highest, and in Ansan, an urban area, the proportion of participants who consumed more than four cups of green tea per week was the highest. In a survey of green tea consumers in Korea, China, and Japan, 216 Koreans answered that health was their motivation for purchasing green tea $(n=80,37.0 \%)$, with the highest proportion of 
participants consuming it at home $(\mathrm{n}=99,45.8 \%)$ or at an office/school $(\mathrm{n}=81,37.5 \%)$ [28] In a result of a survey of consumers' perceptions of green tea in Daegu, Korea, 287 out of 296 participants answered that they drink it because it is good for health [29]. According to the 2008-2019 Community Health Survey conducted by the Korean Statistical Information Service (KOSIS), the health behavior practice rate in Ansan, a large city, was $33.2 \%(n=1822)$, while that in Ansung, a rural area, was 23\% ( $n=908)$ [30]. There was a difference of approximately $10 \%$, depending on the scale of the residential area. This disparity according to a residential area may be due to a difference in the perception of health depending on whether the residential area is urban or rural [31,32]. Additionally, health inequalities depending on the area are one of the socioeconomic factors contributing to the difference in healthy behavior practice rates [31,33]. Differences in health perceptions may have affected the consumption of green tea. The results of this study also showed health inequality in terms of education and income levels, lower consumption of green tea per week, and lower education and income levels. Area, education level, and income level are all socioeconomic factors, indicating that one group has a lower average health level than others [34]. As the standard of living in the 21st century has enhanced overall, the health gap is gradually narrowing, but since socioeconomic differences in Korea have an impact on the risk of abdominal obesity, they are worth paying attention to [34-36].

Drinking and smoking based on the amount of weekly green tea consumption, tended to be inversely associated, depending on sex. The proportion of current drinkers was higher in the group that consumed $\geq 4$ cups/week of green tea than in green tea non-drinkers in men and women. In contrast, the proportion of current drinkers was higher in green tea non-drinkers than in men who consumed $\geq 4$ cups/week of green tea. Alcoholic ethanol oxidation is accompanied by free radical production, and green tea acts as an antioxidant in the liver and serum by preventing ethanol-induced changes [37,38]. This antioxidant effect of green tea is thought to suppress the risk of abdominal obesity in women who consume alcohol. Green tea extracts reduce the level of oxidative stress in smokers [39]. According to cell culture and animal model studies, EGCG, a major polyphenol in green tea, has strong anti-inflammatory and anti-proliferative activities that can selectively inhibit cell growth and induce apoptosis in cancer cells, thus interfering with the adverse effects of tobacco smoke on various organs (bronchial epithelial cells, lungs, and liver) [40-42]. Furthermore, green tea consumption may have a positive effect on the risk of abdominal obesity in both smokers and non-smokers who may be passively exposed to smoke by reducing the level of oxidative stress. In summary, green tea acts as an antioxidant by lowering the oxidative stress caused by smoking and drinking, which reduces their harmful effects and further reduces the risk of abdominal obesity [43].

In this study, higher consumption of green tea was significantly associated with decreased total cholesterol and increased HDL cholesterol levels in women. In men, higher consumption of green tea was associated with significantly increased fasting blood glucose and total cholesterol levels. Many studies have demonstrated that green tea consumption is significantly associated with blood indicators $[15,20,44-46]$. In a case-control study of Sprague-Dawley male rats (180-200 g) in Korea, a combination of GTPs and vitamin C was more effective in reducing blood glucose, insulin resistance, and serum triglycerides than either substance administered alone [47]. In another experimental epidemiological study of male mice aged 4 weeks in Korea, EQS mixed with green tea extract, dietary fiber (polydextrose), and vitamin $\mathrm{C}$ was demonstrated to be highly effective against oxidative stress in obesity [48]. Green tea consumption is related to blood indicators; therefore, it is helpful not only in abdominal obesity but also in cardiovascular diseases such as dyslipidemia, arteriosclerosis, diabetes, and general obesity [15,49-51]. The relationship between total cholesterol and HDL cholesterol levels and green tea intake in women was consistent with previous studies, but the relationship between fasting blood glucose and total cholesterol levels and green tea intake in men was not explained by the results of previous studies [52,53]. 
In women, the higher the amount of green tea consumption, the more significantly lower the systolic and diastolic blood pressures, and in men, the higher the amount of green tea consumption, the more significantly lower the systolic blood pressure. The antioxidant properties of GTPs help to inhibit higher blood pressure and have a positive effect on the treatment of hypertensive patients by lowering blood pressure indicators $[54,55]$. High blood pressure is strongly associated with abdominal obesity, which is a predictor of future hypertension [56]. Both women and men tended to have significantly higher total energy, carbohydrate, and fat intakes and higher green tea consumption. High-carbohydrate and high-fat diets are associated with the risk of abdominal obesity $[57,58]$. In many experimental epidemiological studies that demonstrated the efficacy of green tea, most mice and rats were fed a high-fat diet to induce obesity and a positive effect was observed on blood indicators and lipid metabolism with regard to insulin resistance $[45,49,59,60]$. It has been determined that a high-fat diet intake causes abdominal obesity, but not all dietary fat accumulates as abdominal fat [61,62]. It is also necessary to study the association between the type of dietary fat and green tea consumption through experimental studies on human subjects.

Both women and men tended to have significantly higher dietary fiber and protein intakes with higher green tea consumption. In contrast to total energy, carbohydrate and fat intake, fiber and protein intake were linked to a reduced risk of abdominal obesity [63-66]. In Korea, various experimental epidemiological studies on high-fat diet-induced SpragueDawley rat obesity have confirmed the antioxidant and anti-inflammatory effects of dietary fiber added to green tea, which have a positive effect on abdominal obesity through the reduction of abdominal fat [21,48].

Among the ingredients of green tea, epigallocatechin-3-gallate-rich green tea extract (EGCG), caffeine, and methyl-xanthine have been reported to promote fatty acid oxidation and inhibit fatty synthesis with anti-obesity effects [15,21]. Overall, scientific evidence confirms the positive effects of ingesting green tea catechins, particularly EGCG [67]. Recently, EGCG-containing green tea extract (EGTE), a new functional food material containing more than $97 \%$ of the green tea ingredient EGCG, was developed [68].

Green tea is considered the most representative tea in Korea. Green tea-based foods such as desserts, beverages, and health functional foods have also been widely considered, and events related to green tea have been held continuously in areas where tea is grown. The major strength of this study is the analysis of the risk of abdominal obesity, components of the metabolic syndrome, using data from the KoGES cohort of 10,030 Koreans who routinely consume green tea. With regard to green tea, blood pressure, blood lipids, inflammatory indicators, and obesity have been studied in a variety of ways, but there have been few studies on their association with abdominal obesity. Moreover, this is the first large-scale study to explore the association between green tea consumption and the prevalence of abdominal obesity in the Korean population. Studies on the association between abdominal obesity and several blood indicators support our findings. Recently, as more people have become aware of the efficacy of green tea, several health functional foods using the main ingredients of green tea such as EGCG and EGTE, have been developed. Our findings provide valuable health information that may be of interest to the public.

However, this study has some limitations. Of 3003 women, 1367 were in the abdominal obesity group and 1636 were in the non-abdominal obesity group, creating a similar ratio. However, out of 2875 men, 520 participants were in the abdominal obesity group and 2355 participants were in the non-abdominal obesity group, a difference of four times that may be the cause of disparity in the association between abdominal obesity and green tea consumption between sexes. This is thought to have influenced the lack of statistical power in men. Moreover, a sample from a specific area would not be generalizable in terms of the characteristics of the entire Korean population. Based on the results that differ between men and women, further research on the relationship with green tea intake should be conducted. The effectiveness of green tea depends on the duration and timing of consumption, and the fact that these aspects were not explored in the participants in this study may have affected 
the results [69]. This study investigated only the consumption of green tea beverages, which limits the present study because the type of green tea leaves and ingredients differ depending on the region, and foods using green tea were not considered [70].

\section{Conclusions}

In conclusion, this study found that green tea intake is associated with a reduced risk of abdominal obesity in women. As shown in several studies, the ingredients of green tea are known not only to have anti-obesity effects on blood indicators and lipid profiles, but also to have a beneficial effect on abdominal obesity, a component of metabolic syndrome. Although this study was conducted on middle-aged people, it is expected that adult diseases caused by dietary habits would not be limited to middle-aged people, but would extend many age groups in the future who have an imbalance in nutrient intake and reduced physical activity. Therefore, research that includes various age groups is needed regarding the relationships between green tea, certain nutrients, and physical activities.

Author Contributions: Conceptualization, J.K. and D.S.; data curation, J.K. and D.S.; formal analysis, J.K. and D.S.; investigation, J.K. and D.S.; methodology, J.K. and D.S.; writing-original draft, J.K.; writing-review and editing, J.K. and D.S.; supervision, D.S. All authors have read and agreed to the published version of the manuscript.

Funding: This study received no external funding.

Institutional Review Board Statement: The study protocol was reviewed and approved by the Institutional Review Board (IRB) of Inha University on 31 January 2020 (IRB No. 200129-1A).

Informed Consent Statement: The KoGES study was reviewed and approved by the Institutional Review Board of the Korea Centers for Disease Control and Prevention. All participants were voluntarily enrolled in the study and provided written informed consent. All study methods and protocols were conducted in accordance with relevant institutional guidelines and regulations.

Data Availability Statement: The data underlying the results of our study are not publicly available because of KoGES data policy. Data are available from the Division of Genetic Epidemiology and Health Index, NIH, Korea Centers for Disease Control and Prevention, for researchers who meet the criteria for access to confidential data.

Acknowledgments: This study was conducted with biosources from the National Biobank of Korea, Centers for Disease Control and Prevention, Republic of Korea (KBN-2020-016).

Conflicts of Interest: The authors declare no conflict of interest.

\section{References}

1. Ritchie, S.; Connell, J. The link between abdominal obesity, metabolic syndrome and cardiovascular disease. Nutr. Metab. Cardiovasc. Dis. 2007, 17, 319-326. [CrossRef] [PubMed]

2. National Health Insurance Service. National Health Screening Statistical Yearbook 2019; National Health Insurance Service: Wonju, Gangwon-do, Korea, 2019; Available online: https://www.nhis.or.kr/nhis/together/wbhaec07000m01.do?mode= view\&articleNo=10803730 (accessed on 2 March 2021).

3. Dagenais, G.R.; Yi, Q.; Mann, J.F.; Bosch, J.; Pogue, J.; Yusuf, S.; HOPE Study Investigators. Prognostic impact of body weight and abdominal obesity in women and men with cardiovascular disease. Am. Heart J. 2005, 149, 54-60. [CrossRef] [PubMed]

4. Postorino, M.; Marino, C.; Tripepi, G.; Zoccali, C.; CREDIT Working Group. Abdominal obesity and all-cause and cardiovascular mortality in end-stage renal disease. J. Am. Coll. Cardiol. 2009, 53, 1265-1272. [CrossRef]

5. Pischon, T.; Boeing, H.; Hoffmann, K.; Bergmann, M.; Schulze, M.B.; Overvad, K.; Van der Schouw, Y.; Spencer, E.; Moons, K.; Tjønneland, A. General and abdominal adiposity and risk of death in Europe. N. Engl. J. Med. 2008, 359, 2105-2120. [CrossRef] [PubMed]

6. Lee, S.H.; Jo, B.N.; Hong, Y.J.; Lee, M.S.; Joo, S.S.; Kim, D.M.; Jin, D.G.; No, S.L. Effects of green tea, cocoon hydrolysates and capsulated components mixture on body fat and serum lipid composition in college women in terms of supplemented periods. $J$. Korean Soc. Food Sci. Nutr. 2003, 32, 715-722.

7. Carey, D.G. Abdominal obesity. Curr. Opin. Lipidol. 1998, 9, 35-40. [CrossRef]

8. Björntorp, P. Do stress reactions cause abdominal obesity and comorbidities? Obes. Rev. 2001, 2, 73-86. [CrossRef]

9. Lee, J.W.; Yoo, S.Y.; Yang, S.Y.; Kim, H.; Cho, S.K. Effect of an abdominal obesity management program on dietary intake, stress index, and waist to hip ratio in abdominally obese women. Korean J. Food Nutr. 2012, 45, 127-139. [CrossRef] 
10. Na, D.W.; Jeong, E.; Noh, E.K.; Chung, J.S.; Choi, C.H.; Park, J. Dietary factors and metabolic syndrome in middle-aged men. J. Agric. Med. Community Health 2010, 35, 383-394. [CrossRef]

11. Romaguera, D.; Ängquist, L.; Du, H.; Jakobsen, M.U.; Forouhi, N.G.; Halkjær, J.; Feskens, E.J.; Masala, G.; Steffen, A.; Palli, D. Food composition of the diet in relation to changes in waist circumference adjusted for body mass index. PLoS ONE 2011, 6, e23384. [CrossRef]

12. Rains, T.M.; Agarwal, S.; Maki, K.C. Antiobesity effects of green tea catechins: A mechanistic review. J. Nutr. Biochem. 2011, 22, 1-7. [CrossRef] [PubMed]

13. Jung, Y.H.; Han, S.H.; Shin, M.K. Effects of green and black Korean teas on lipid metabolism in diet-induced hyperlipidemic rats. J. East Asian Soc. Diet. Life. 2006, 16, 550-558

14. Lee, M.Y.; Yeom, Y.G. Antihyperlipidemic effects of fried beancurd containing powdered green tea in rats. J. Korea Acad. Ind. Coop. Soc. 2004, 5, 546-551.

15. Nagao, T.; Hase, T.; Tokimitsu, I. A green tea extract high in catechins reduces body fat and cardiovascular risks in humans. Obesity 2007, 15, 1473-1483. [CrossRef]

16. Sayama, K.; Lin, S.; Zheng, G.; Oguni, I. Anti-obesity effects of three major components of green tea, catechins, caffeine and theanine, in mice. In Vivo 2004, 18, 55-62.

17. Maki, K.C.; Reeves, M.S.; Farmer, M.; Yasunaga, K.; Matsuo, N.; Katsuragi, Y.; Komikado, M.; Tokimitsu, I.; Wilder, D.; Jones, F. Green tea catechin consumption enhances exercise-induced abdominal fat loss in overweight and obese adults. J. Nutr. 2009, 139, 264-270. [CrossRef]

18. Huang, J.; Zhang, Y.; Zhou, Y.; Zhang, Z.; Xie, Z.; Zhang, J.; Wan, X. Green tea polyphenols alleviate obesity in broiler chickens through the regulation of lipid-metabolism-related genes and transcription factor expression. J. Agric. Food Chem. 2013, 61, 8565-8572. [CrossRef]

19. Wang, H.; Wen, Y.; Du, Y.; Yan, X.; Guo, H.; Rycroft, J.A.; Boon, N.; Kovacs, E.M.; Mela, D.J. Effects of catechin enriched green tea on body composition. Obesity 2010, 18, 773-779. [CrossRef]

20. Choi, M.J.; Kim, S.H. Effects of green tea powder supplementation on blood glucose and lipid concentrations in rats with streptozotocin-induced diabetes. J. East. Asian Soc. Diet. Life 2010, 20, 46-53.

21. Lee, S.J.; Kim, K.R.; Kim, H.T.; Hong, J.H. Effects of catechin on lipid composition and adipose tissue in obese rats fed high fat diet. J. Korean Soc. Food Sci. Nutr. 2007, 36, 540-547.

22. Kim, Y.J.; Han, B.G.; KoGES Group. Cohort profile: The Korean Genome and Epidemiology Study (KoGES) consortium. Int. J. Epidemiol. 2017, 46, e20. [CrossRef]

23. Moon, S.; Oh, C.M.; Choi, M.K.; Park, Y.K.; Chun, S.K.; Choi, M.K.; Yu, J.M.; Yoo, H.J. The influence of physical activity on risk of cardiovascular disease in people who are obese but metabolically healthy. PLoS ONE 2017, 12, e0185127. [CrossRef] [PubMed]

24. Lee, K.W.; Shin, D. Positive association between dietary acid load and future insulin resistance risk: Findings from the Korean Genome and Epidemiology Study. Nutr. J. 2020, 19, 1-11. [CrossRef] [PubMed]

25. Kim, J.S.; Kim, Y.J.; Ahn, Y.O.; Paik, H.Y.; Aim, Y.J.; Tokudome, Y.; Hamajima, N.; Inouc, M.; Tajima, K.; Ahn, Y.J. Development of a food frequency questionnaire in Koreans. Asia Pac. J. Clin. Nutr. 2003, 12, 243-250.

26. Korea Centers for Disease Control and Prevention. Korean Genome and Epidemiology Study (KoGES) Food Frequency Questionnaire guideline. Available online: https://www.kdca.go.kr/contents.es?mid=a40504040200 (accessed on 13 January 2022).

27. Bulló, M.; Garcia-Aloy, M.; Martínez-González, M.A.; Corella, D.; Fernández-Ballart, J.D.; Fiol, M.; Gómez-Gracia, E.; Estruch, R.; Ortega-Calvo, M.; Francisco, S. Association between a healthy lifestyle and general obesity and abdominal obesity in an elderly population at high cardiovascular risk. Prev. Med. 2011, 53, 155-161. [CrossRef] [PubMed]

28. Park, J.H.; Cho, M.H.; Lee, K.H. A cross-cultural study of Korean Chinese Japanese consumer perceptions about green tea quality attributes and their consumption behavior. Int. J. Tour. Hosp. Res. 2018, 32, 251-266.

29. Lee, J.O.; Yoo, K.H. The perception of the green tea among consumers in Taegu area. J. Life Sci. Res. 2009, 35, 191-215.

30. Korean Statistical Information Service (KOSIS). Healthy Living Practice Rate by City, County, and District. Available online: https:/ / kosis.kr/statHtml/statHtml.do?orgId=177\&tblId=DT_117075_H_HEALTHY\&conn_path=I2 (accessed on 9 April 2021).

31. Hartley, D. Rural health disparities, population health, and rural culture. Am. J. Public Health 2004, 94, 1675-1678. [CrossRef]

32. Ogunbodede, E.O.; Kida, I.A.; Madjapa, H.; Amedari, M.; Ehizele, A.; Mutave, R.; Sodipo, B.; Temilola, S.; Okoye, L. Oral health inequalities between rural and urban populations of the African and Middle East region. Adv. Dent. Res. 2015, 27, 18-25. [CrossRef]

33. Ferrer, R.A.; Klein, W.M. Risk perceptions and health behavior. Curr. Opin. Psychiatry 2015, 5, 85-89. [CrossRef]

34. Lee, M.S. Health inequalities among Korean adults-Socioeconomic status and residential area differences. Korean J. Sociol. 2005, 39, 183-209.

35. Lee, K.O.; Yoon, H.S. Relationship between inequalities in health and inequalities in socioeconomic status. J. Korean Acad. Community Health Nurs. 2001, 12, 609-619.

36. Yoon, Y.S.; Oh, S.W.; Park, H.S. Socioeconomic status in relation to obesity and abdominal obesity in Korean adults: A focus on sex differences. Obesity 2006, 14, 909-919. [CrossRef]

37. Skrzydlewska, E.; Ostrowska, J.; Stankiewicz, A.; Farbiszewski, R. Green tea as a potent antioxidant in alcohol intoxication. Addict. Biol. 2002, 7, 307-314. [CrossRef] [PubMed] 
38. Arteel, G.E.; Uesugi, T.; Bevan, L.N.; Gäbele, E.; Wheeler, M.D.; McKim, S.E.; Thurman, R.G. Green tea extract protects against early alcohol-induced liver injury in rats. Biol. Chem. 2002, 383, 663-670. [CrossRef]

39. Holzer, N.; Braun, K.F.; Ehnert, S.; Egaña, J.T.; Schenck, T.L.; Buchholz, A.; Schyschka, L.; Neumaier, M.; Benzing, S.; Stöckle, U. Green tea protects human osteoblasts from cigarette smoke-induced injury: Possible clinical implication. Langenbecks Arch. Surg. 2012, 397, 467-474. [CrossRef] [PubMed]

40. Syed, D.; Afaq, F.; Kweon, M.; Hadi, N.; Bhatia, N.; Spiegelman, V.; Mukhtar, H. Green tea polyphenol EGCG suppresses cigarette smoke condensate-induced NF-к B activation in normal human bronchial epithelial cells. Oncogene 2007, 26, 673-682. [CrossRef]

41. Leanderson, P.; Faresjö, Å.O.; Tagesson, C. Green tea polyphenols inhibit oxidant-induced DNA strand breakage in cultured lung cells. Free Radic. Biol. Med. 1997, 23, 235-242. [CrossRef]

42. Gao, J.; Tang, H.; Zhao, B. Toxicologic damage of gas phase cigarette smoke on cells and the protective effect of green tea polyphenols. Res. Chem. Intermed. 2001, 27, 269-279. [CrossRef]

43. Nakachi, K.; Matsuyama, S.; Miyake, S.; Suganuma, M.; Imai, K. Preventive effects of drinking green tea on cancer and cardiovascular disease: Epidemiological evidence for multiple targeting prevention. Biofactors 2000, 13, 49-54. [CrossRef]

44. Onakpoya, I.; Spencer, E.; Heneghan, C.; Thompson, M. The effect of green tea on blood pressure and lipid profile: A systematic review and meta-analysis of randomized clinical trials. Nutr. Metab. Cardiovasc. Dis. 2014, 24, 823-836. [CrossRef] [PubMed]

45. Bose, M.; Lambert, J.D.; Ju, J.; Reuhl, K.R.; Shapses, S.A.; Yang, C.S. The major green tea polyphenol, (-)-epigallocatechin-3-gallate, inhibits obesity, metabolic syndrome, and fatty liver disease in high-fat-fed mice. J. Nutr. 2008, 138, 1677-1683. [CrossRef] [PubMed]

46. Chen, I.J.; Liu, C.Y.; Chiu, J.P.; Hsu, C.H. Therapeutic effect of high-dose green tea extract on weight reduction: A randomized, double-blind, placebo-controlled clinical trial. Clin. Nutr. 2016, 35, 592-599. [CrossRef]

47. Lee, B.R.; Yang, H.; Park, P.S. Effects of green tea polyphenol and vitamin C on type 2 diabetic rats induced by low dose streptozotocin following high fat diet. Korean J. Food Nutr. 2016, 45, 167-173. [CrossRef]

48. Kim, J.D.; Lee, B.I.; Jeon, Y.H.; Park, J.P.; Kim, H.R.; Lim, B.W. Anti-oxidative and anti-inflammatory effects of green tea mixture and dietary fiber on liver of high fat diet-induced obese rats. Korean J. Med. Crop. Sci. 2010, 18, 224-230.

49. Chen, Y.K.; Cheung, C.; Reuhl, K.R.; Liu, A.B.; Lee, M.J.; Lu, Y.P.; Yang, C.S. Effects of green tea polyphenol (-)-epigallocatechin3-gallate on newly developed high-fat/Western-style diet-induced obesity and metabolic syndrome in mice. J. Agr. Food Chem. 2011, 59, 11862-11871. [CrossRef] [PubMed]

50. Senger, A.V.; Schwanke, C.; Gomes, I.; Gottlieb, M.G.V. Effect of green tea (Camellia sinensis) consumption on the components of metabolic syndrome in elderly. J. Nutr. Health Aging 2012, 16, 738-742. [CrossRef]

51. Bhardwaj, P.; Khanna, D. Green tea catechins: Defensive role in cardiovascular disorders. Chin. J. Nat. Med. 2013, 11, 345-353. [CrossRef]

52. Yokozawa, T.; Nakagawa, T.; Kitani, K. Antioxidative activity of green tea polyphenol in cholesterol-fed rats. J. Agric. Food Chem. 2002, 50, 3549-3552. [CrossRef]

53. Liu, K.; Zhou, R.; Wang, B.; Chen, K.; Shi, L.-Y.; Zhu, J.-D.; Mi, M.-T. Effect of green tea on glucose control and insulin sensitivity: A meta-analysis of 17 randomized controlled trials. Am. J. Clin. Nutr. 2013, 98, 340-348. [CrossRef]

54. Negishi, H.; Xu, J.-W.; Ikeda, K.; Njelekela, M.; Nara, Y.; Yamori, Y. Black and green tea polyphenols attenuate blood pressure increases in stroke-prone spontaneously hypertensive rats. J. Nutr. 2004, 134, 38-42. [CrossRef]

55. Bogdanski, P.; Suliburska, J.; Szulinska, M.; Stepien, M.; Pupek-Musialik, D.; Jablecka, A. Green tea extract reduces blood pressure, inflammatory biomarkers, and oxidative stress and improves parameters associated with insulin resistance in obese, hypertensive patients. Nutr. Res. Rev. 2012, 32, 421-427. [CrossRef]

56. Chuang, S.Y.; Chou, P.; Hsu, P.F.; Cheng, H.M.; Tsai, S.T.; Lin, I.F.; Chen, C.H. Presence and progression of abdominal obesity are predictors of future high blood pressure and hypertension. Am. J. Hypertens. 2006, 19, 788-795. [CrossRef]

57. Kuo, L.E.; Czarnecka, M.; Kitlinska, J.B.; Tilan, J.U.; Kvetňanský, R.; Zukowska, Z. Chronic stress, combined with a high-fat/highsugar diet, shifts sympathetic signaling toward neuropeptide $\mathrm{Y}$ and leads to obesity and the metabolic syndrome. Ann. N. Y. Acad. Sci. 2008, 1148, 232. [CrossRef]

58. Han, D.H.; Hansen, P.A.; Host, H.H.; Holloszy, J.O. Insulin resistance of muscle glucose transport in rats fed a high-fat diet: A reevaluation. Diabetes 1997, 46, 1761-1767. [CrossRef] [PubMed]

59. Xu, Y.; Zhang, M.; Wu, T.; Dai, S.; Xu, J.; Zhou, Z. The anti-obesity effect of green tea polysaccharides, polyphenols and caffeine in rats fed with a high-fat diet. Food Funct. 2015, 6, 296-303. [CrossRef]

60. Vessby, B. Dietary fat and insulin action in humans. Br. J. Nutr. 2000, 83, S91-S96. [CrossRef] [PubMed]

61. Suara, S.B.; Siassi, F.; Saaka, M.; Foroshani, A.R.; Asadi, S.; Sotoudeh, G. Dietary fat quantity and quality in relation to general and abdominal obesity in women: A cross-sectional study from Ghana. Lipids Health Dis. 2020, 19, 1-13. [CrossRef]

62. Grundy, S.M.; Abate, N.; Chandalia, M. Diet composition and the metabolic syndrome: What is the optimal fat intake? Am. J. Med. 2002, 113, 25-29. [CrossRef]

63. SHARE and SHARE-AP Investigators. Protein intake is inversely associated with abdominal obesity in a multi-ethnic population. J. Nutr. 2005, 135, 1196-1201. [CrossRef] [PubMed]

64. Fujii, H.; Iwase, M.; Ohkuma, T.; Ogata-Kaizu, S.; Ide, H.; Kikuchi, Y.; Idewaki, Y.; Joudai, T.; Hirakawa, Y.; Uchida, K. Impact of dietary fiber intake on glycemic control, cardiovascular risk factors and chronic kidney disease in Japanese patients with type 2 diabetes mellitus: The Fukuoka Diabetes Registry. Nutr. J. 2013, 12, 1-8. [CrossRef] [PubMed] 
65. Loenneke, J.P.; Wilson, J.M.; Manninen, A.H.; Wray, M.E.; Barnes, J.T.; Pujol, T.J. Quality protein intake is inversely related with abdominal fat. Nutr. Metab. 2012, 9, 1-3. [CrossRef] [PubMed]

66. Arciero, P.J.; Ormsbee, M.J.; Gentile, C.L.; Nindl, B.C.; Brestoff, J.R.; Ruby, M. Increased protein intake and meal frequency reduces abdominal fat during energy balance and energy deficit. Obesity 2013, 21, 1357-1366. [CrossRef] [PubMed]

67. Thielecke, F.; Boschmann, M. The potential role of green tea catechins in the prevention of the metabolic syndrome- $-\mathrm{A}$ review. Phytochemistry 2009, 70, 11-24. [CrossRef]

68. Cheon, S.I.; Heo, E.J.; Yoon, M.J.; Choi, S.U.; Ryu, G.S.; Ryu, S.Y. Evaluation for long-term stability of EGCG rich green tea extract (EGTE). Kor. J. Pharmacogn. 2018, 49, 328-335.

69. Nho, H.S.; Choi, S.K.; Lim, K.W. The effects of exercise and diet therapy on visceral fat area of the obese women. Korean J. Sports Med. 1999, 38, 428-438.

70. Ahmad, T.; Lee, I.M.; Paré, G.; Chasman, D.I.; Rose, L.; Ridker, P.M.; Mora, S. Lifestyle interaction with fat mass and obesityassociated (FTO) genotype and risk of obesity in apparently healthy US women. Diabetes Care 2011, 34, 675-680. [CrossRef] 